Artículo Original/ Original Article

http://dx.doi.org/10.18004/mem.iics/1812-9528/2021.019.02.21

\title{
Características clínico-demográficas de pacientes con ceguera irreversible en el Servicio de Oftalmología General de la Clínica Belén, Coronel Oviedo, Paraguay desde febrero del 2018 a febrero del 2019
}

\author{
Andrea Cabrera-Gonzales , Amanda Guerrero ${ }^{D}$, Margarita Samudio \\ Rainald Duerksen iD \\ ${ }^{1}$ Fundación Visión. Fernando de la Mora, Paraguay
}

Cómo referenciar este artículo/ How to reference this article:

\begin{abstract}
Cabrera-Gonzales A, Guerrero A, Samudio M, Duerksen R. Características clínico-demográficas de pacientes con ceguera irreversible en el Servicio de Oftalmología General de la Clínica Belén, Coronel Oviedo, Paraguay desde febrero del 2018 a febrero del 2019. Mem. Inst. Investig. Cienc. Salud. 2021; 19(2): $21-31$
\end{abstract}

\begin{abstract}
RES U ME N
Según la Organización Mundial de la Salud, la ceguera está definida como la agudeza visual inferior a $3 / 60(20 / 400)$ en el mejor ojo y un campo visual menor a $10^{\circ}$ desde el punto central de fijación. El objetivo de este trabajo fue describir las características clínico demográficas de pacientes con diagnóstico de ceguera irreversible atendidos en el Servicio de Oftalmología General de la Clínica Belén - Coronel Oviedo (Paraguay). Estudio observacional, descriptivo y retrospectivo de corte transversal. Se realizó la revisión retrospectiva de las historias clínicas de los pacientes con diagnóstico de ceguera irreversible atendidos en el Servicio de Oftalmología General de la Clínica Belén, entre el 1 de febrero 2018 y el 28 de febrero 2019. Se analizaron la edad, sexo, escolaridad, procedencia, agudeza visual, comorbilidades y etiología de la deficiencia visual. El análisis estadístico fue mediante el cálculo de frecuencias absolutas y relativas para las variables cualitativas, y el promedio y desviación estándar para las cuantitativas. Se estudiaron 78 pacientes, con predominio del sexo masculino $(56,4 \%)$, edad de 71 a 95 años $(43,6 \%)$ y primaria incompleta $(41 \%)$. La hipertensión arterial $(55,6 \%)$ fue la principal comorbilidad y glaucoma $(43,6 \%)$ la etiología de ceguera más frecuente. El glaucoma fue más frecuente en varones que en mujeres ( $59 \%$ vs $32 \%$ ) y en pacientes mayores de 50 años de edad (50\%). En esta serie, los pacientes presentaron ceguera irreversible bilateral, en mayor frecuencia en varones, mayores de 50 años de edad, hipertensión como comorbilidad y glaucoma como etiología más frecuente.
\end{abstract}

Palabras clave: características clínico-demográficas, agudeza visual, ceguera, Paraguay (DeCS).

\section{Clinical and demographic characteristics of patients with irreversible blindness at the General Ophthalmology Service of the "Clínica Belén", Coronel Oviedo, Paraguay from February 2018 to February 2019}

According to the World Health Organization, blindness is defined as visual acuity less than $3 / 60(20 / 400)$ in the better eye and a visual field less than $10^{\circ}$ from the central 
fixation point. The objective was to describe the clinical demographic characteristics of patients with a diagnosis of irreversible blindness treated at the General Ophthalmology Service of the "Clínica Belén" - "Coronel Oviedo" (Paraguay). This was an observational, descriptive and retrospective cross-sectional study. A retrospective review of the medical records of patients with a diagnosis of irreversible blindness treated at the General Ophthalmology Service of the "Clínica Belén" between February 1, 2018 and February 28, 2019 was carried out. Age, sex, education, origin, visual acuity, comorbidities and etiology of visual impairment were analyzed. The statistical analysis was through the calculation of absolute and relative frequencies, as well as the average and standard deviation. Seventy eight patients were studied, with a slight predominance of males (56.4\%), aged 71 to 95 years $(43.6 \%)$, incomplete primary school (41\%) and from "Coronel Oviedo" (25.6\%) and surroundings. Arterial hypertension $(55.6 \%)$ was the main comorbidity and glaucoma $(43.6 \%)$ the most frequent blindness etiology. Glaucoma was more common in male patients than female patients (59\% and 32\%, respectively) and in patients older than 50 years of age $(50 \%)$. In this series, patients presented bilateral irreversible blindness, more frequent in men, older than 50 years of age, hypertension as comorbidity and glaucoma as the most frequent etiology.

Keywords: Clinical-demographic characteristics, visual acuity, blindness, Paraguay (DeCS).

\section{INTRODUCCIÓN}

En un mundo construido sobre la capacidad de ver, la visión se posiciona como uno de los sentidos más importantes de la población ${ }^{(1)}$, ya que además de resguardar los niveles de generalización sociofamiliar, permite la preservación de la autonomía personal y de la agudeza mental ${ }^{(2)}$. Sin embargo, los esfuerzos mundiales para promover la salud ocular aún son deficientes y predicen un incremento de la tendencia epidemiológica de ciegos, junto al crecimiento poblacional, envejecimiento y a los cambios de estilos de vida ${ }^{(1,3)}$.

Según la Organización Mundial de la Salud (OMS), la ceguera está definida como la agudeza visual inferior a $3 / 60(20 / 400)$ en el mejor ojo $^{(4)}$ y un campo visual menor a $10^{\circ}$ desde el punto central de fijación ${ }^{(5)}$.

A nivel mundial aproximadamente dos mil millones de personas presentan deterioro visual, de las cuales alrededor de la mitad, han podido ser prevenibles o haber llevado algún tipo de tratamiento. De estos mil millones, aquellos que tienen deterioro grave de la visión distante, la catarata afecta aproximadamente a 94 millones de personas, el glaucoma a 7,7 millones, mientras que los errores refractarios no corregidos afectan a 88.4 millones; y el deterioro de la visión cercana afecta a 826 millones de personas aproximadamente ${ }^{(4)}$.

Paraguay no es ajena a esta problemática, ya que fue el primer país de América en realizar una evaluación rápida de los servicios de cirugía de cataratas (RACS-1999), con el objeto de reducir la tendencia epidemiológica de ciegos $(3.1 \%$ de ciegos bilaterales en 1999), concientizar a la población y establecer un plan nacional de lucha contra la ceguera ${ }^{(6,7)}$. Bajo ese contexto, en el año 2003, se creó el primer Comité Nacional de Visión 2020 (CONAVIP-Paraguay) de América Latina, que no es más que una plataforma de diálogo y consenso sobre el cuidado de la visión, liderada por el país mediterráneo y encargada de coordinar y recopilar información estadística anual sobre las consultas y cirugías oftalmológicas ${ }^{(6)}$.

En 2003, se estimó la carga de ceguera por catarata en personas iguales o mayores de 50 años en 40 conglomerados de toda la población de Paraguay, donde, se encontró una prevalencia de ceguera bilateral ajustada por edad y sexo de $3.14 \%$, mientras que la prevalencia ajustada de ceguera bilateral por catarara fue de $2.1 \%$, siendo esta patología la principal causa de ceguera bilateral en dicho grupo etareo ${ }^{(8)}$. En la Evaluación Rápida de Ceguera Evitable (ERCE), se resume la prevalencia de la patología ajustada por edad y sexo, entre los años 1999-2011: donde se evidenció que la prevalencia de ceguera bilateral en 1999 fue de $3.1 \%$ mientras que en el 2011 disminuyó a $1.1 \%$, luego al realizar dicho ajuste por cataratas se identificó que en 1999 la prevalencia fue de $2.0 \%$ y en el 2011 fue de $0.3 \%{ }^{(6)}$. 
Adicionalmente, existe la ceguera legal la cual es definida según estándares de cada país, por ejemplo, en Estados Unidos los National Institutes of Health (NIH) reportaron en el 2015, 1 millón de individuos legalmente ciegos (visión $\leq 20 / 200$ ), con mayor preponderancia en los ancianos del sexo femenino y de raza blanca no hispana. Así mismo, es necesario destacar las proyecciones realizadas para el año 2050, que auguran un incremento de $21 \%$ por cada década y un total de 2 millones de personas con ceguera legal ${ }^{(9)}$. América Latina y El Caribe exteriorizan un escenario similar, ya que se estima aproximadamente 2.3 millones de individuos con ceguera, posicionando como causas principales a la catarata (40-76\%), defectos refractivos no corregidos $(18-50 \%)$ y a patologías del segmento posterior $(2-23 \%)^{(10)}$. Y de manera más específica en Bolivia al realizar la Encuesta Nacional de Ceguera y Deficiencia Visual Evitable en el 2015, identificaron que la prevalencia de ceguera era de 3.3\%, siendo más frecuente en el sexo femenino (3.7\%), cuyas principales causas de esta discapacidad visual eran la catarata $(66.7 \%)$ y el glaucoma $(7.6 \%)^{(11)}$.

La Fundación Visión fue creada en 1999, es una institución líder en la prevención de ceguera y servicios de salud ocular de alta tecnología, además encargada de formar profesionales oftalmólogos enfocados en la salud comunitaria. Esta Fundación atiende alrededor de 70 mil pacientes por año, entre los cuales 8500 se someten a estudios oftalmológicos, 3000 a cirugías oculares y 2500 a procedimientos ambulatorios. En el 2002 se inauguró la Clínica Belén en Coronel Oviedo, ubicada en un área rural, específicamente en la Compañía Espinillo Km 127; que entre febrero del 2018 y febrero del 2019 registró un total de 12000 consultas oftalmológicas, entre las cuales 78 estuvieron relacionadas con diagnósticos de ceguera irreversible ${ }^{(12)}$.

Debido a la existencia de gran número de personas ciegas en el mundo, siendo en su mayoría casos prevenibles o curables $(80 \%)$, este estudio pretende identificar la cantidad de personas con ceguera irreversible y sus principales causas, con la finalidad de tomar medidas correctivas, e influya en la reducción de los casos en Paraguay, mejorando la atención y rehabilitación visual. No se cuenta con un registro epidemiológico-clínico, que permita establecer con certeza cada una de las particularidades de la ceguera. Por ello, se torna imprescindible la ejecución de una investigación que permita establecer con certeza dichas características, ya que su constitución permitiría adoptar medidas correctivas y reducir la tendencia epidemiológica de ciegos en el territorio nacional.

La presente investigación tiene gran relevancia puesto que brindará información actual sobre las características demográficas de los pacientes que padecen ceguera irreversible, que fueron evaluados en la Clínica Belén, así mismo se identificarán las causas de dicha ceguera, lo cual será de gran utilidad para dar un mayor alcance sobre los posibles programas o estrategias que se puedan realizar en esta población, donde los pobladores que padezcan de alguna causa de ceguera puedan tener acceso a un posible tratamiento, disminuyendo la progresión de la ceguera o logrando una mejoría en su calidad de vida.

Finalmente debido a que en la región Cnel. Oviedo Compañía Espinillo no se ha analizado e identificado la cantidad de personas con ceguera irreversible, los datos obtenidos en esta investigación aportarán información estadística que servirá como base para la elaboración de otros estudios bajo la misma línea de investigación en la región y para otras realidades poblacionales. El objetivo del estudio es describir las características clínicas y demográficas de los pacientes con diagnóstico de ceguera irreversible atendidos en el Servicio de Oftalmología General de la Clínica Belén, en el periodo de 1 de febrero del 2018 al 28 de febrero del 2019.

\section{MATERIALES Y MÉTODOS \\ Diseño y lugar de estudio}

Observacional, descriptivo, de corte transverso. Los datos correspondieron a los pacientes atendidos en el Servicio de Oftalmología General de la Clínica Belén, el cual se encuentra ubicado en Cnel. Oviedo Compañía Espinillo km 127 - Paraguay.

\section{Población estudiada}

En el periodo de estudio de la presente investigación (1 de febrero del 2018 al 28 de febrero del 2019) se realizó la consulta oftalmológica de 12000 pacientes en el 
Servicio de Oftalmología General de la Clínica Belén. En total se estudiaron 78 pacientes, con diagnóstico de ceguera irreversible; atendidos en el Servicio de Oftalmología General de la Clínica Belén.

\section{Criterios de inclusión}

Pacientes de ambos sexos, de todas las edades, con diagnóstico de ceguera irreversible ( $\mathrm{AV} \leq 20 / 400$ ) que no mejoraron con el tratamiento clínico o quirúrgico.

\section{Criterios de exclusión}

Pacientes que después del tratamiento clínico o quirúrgico tuvieron una agudeza visual, mejor a 20/200.

\section{Muestreo}

Muestreo de tipo no probabilístico por conveniencia.

\section{Reclutamiento}

Se solicitó los permisos a las entidades correspondientes para la ejecución de este estudio; las historias clínicas que cumplieron con los criterios de inclusión y de exclusión, fueron seleccionadas para transcribir los datos a analizar a la ficha de recolección elaborada por la investigadora, la cual cada una de ellas fue codificada para identificar la información de cada paciente, sin necesidad de colocar sus nombres o apellidos.

\section{Variables}

Características demográficas: sexo, edad, escolaridad y procedencia

Características clínicas: comorbilidades, evaluación visual, medición de la agudeza visual mediante la cartilla de Snellen, percepción de la luz y movimiento de manos, etiología de la ceguera

\section{Plan de tabulación y análisis estadísticos}

La base de datos en Excel fue analizada mediante EpiInfo v. 7.0 (CDC, Atlanta). Se calcularon las frecuencias absolutas y las frecuencias relativas porcentual de las variables cualitativas, promedio y DE para las variables cuantitativas. Los resultados se presentaron mediante tablas simples y tablas de doble entrada, además se realizaron gráficos (diagrama de barras, diagrama de barras agrupadas y diagrama de pirámide) que fueron elaborados en Microsoft Excel 2016. Para establecer asociación entre las variables se utilizó la prueba Chi cuadrado a un nivel de significancia de 0,05 .

\section{Asuntos éticos}

El estudio se realizó bajo la Declaración de Helsinki de la Asociación Médica Mundial. Última revisión en la asamblea número 64a Asamblea General, Fortaleza, Brasil, octubre $2013^{(59)}$. El estudio presentado se clasifica dentro de lo que comprendería la Investigación sin riesgo, es un estudio que emplea técnicas y métodos de investigación documental retrospectivos y no se realiza ninguna intervención o modificación intencionada en las variables fisiológicas, psicológicas y sociales de los individuos que participan en el estudio. Este estudio al ser de tipología retrospectiva, la información fue obtenida de las historias clínicas de los pacientes en estudio, por lo tanto, no se tuvo contacto directo con ellos. La información tomada de las historias clínicas fue vaciada en fichas de recolección, las cuales fueron codificadas para poder ser identificadas, de esta manera se evitó colocar nombres o apellidos de los pacientes, resguardando así su identidad.

\section{RESULTADOS}

\section{Características generales}

De los 78 pacientes incluidos en el estudio, $44(56,4 \%)$ fueron del sexo masculino. La edad promedio fue $62,7 \pm 20,2$ años y el $43,6 \%$ pertenecieron al grupo etario de 71 a 95 años. El $41 \%$ de los pacientes tenían la primaria incompleta, el 26,5\% eran procedentes de Coronel Oviedo, igual proporción $(5,1 \%)$ de Caaguazú, Campo 9, y Villarrica, entre otros (Tabla 1). 
Tabla 1. Características demográficas de los pacientes con diagnóstico de ceguera irreversible atendidos en el Servicio de Oftalmología General de la Clínica Belén de Coronel Oviedo de 1 de febrero del 2018 al 28 de febrero del 2019

\begin{tabular}{lrr}
\hline Características generales & $\mathbf{n}$ & $\mathbf{\%}$ \\
\hline Sexo & 34 & $43,6 \%$ \\
Femenino & 44 & $56,4 \%$ \\
Masculino & & \\
Grupo etario & 4 & $5,1 \%$ \\
$<18$ años & 14 & $17,9 \%$ \\
18 a 50 años & 26 & $33,3 \%$ \\
51 a 70 años & 34 & $43,6 \%$ \\
71 a 95 años & & \\
Escolaridad & 7 & $9,0 \%$ \\
Sin escolaridad & 32 & $41,0 \%$ \\
Primaria incompleta & 7 & $9,0 \%$ \\
Primaria completa & 12 & $15,4 \%$ \\
Secundaria incompleta & 4 & $5,1 \%$ \\
Secundaria completa & 1 & $1,3 \%$ \\
Universitaria & 15 & $19,2 \%$ \\
Desconocida & & \\
Procedencia & 20 & $25,6 \%$ \\
Coronel Oviedo & 4 & $5,1 \%$ \\
Caaguazú & 4 & $5,1 \%$ \\
Campo 9 & 4 & $5,1 \%$ \\
Villarrica & 2 & $2,6 \%$ \\
Juan Emiliano O'Leary & 2 & $2,6 \%$ \\
Otros Estanislao & 7 & $9,0 \%$ \\
Desconocida & 35 & $44,9 \%$ \\
\hline Total & $\mathbf{7 8}$ & $\mathbf{1 0 0} \%$ \\
\hline
\end{tabular}

\section{Características Clínicas}

Respecto a la agudeza visual, $27(34,6 \%)$ pacientes no tenían percepción de luz en el ojo derecho y $31(39,7 \%)$ en el ojo izquierdo (Tabla 2$)$.

Tabla 2. Agudeza visual de los pacientes con diagnóstico de ceguera irreversible atendidos en el Servicio de Oftalmología General de la Clínica Belén, de Coronel Oviedo de 1 de febrero del 2018 al 28 de febrero del 2019

\begin{tabular}{lcc}
\hline Agudeza visual & $\mathbf{n}$ & $\mathbf{\%}$ \\
\hline Ojo derecho & & \\
$20 / 400$ & 1 & $1,3 \%$ \\
$20 / 800$ & 3 & $3,8 \%$ \\
$20 / 1200$ & 12 & $15,4 \%$ \\
MM & 21 & $26,9 \%$ \\
PL & 14 & $17,9 \%$ \\
NPL & 27 & $34,6 \%$ \\
Ojo izquierdo & & \\
$20 / 400$ & 4 & $5,1 \%$ \\
$20 / 800$ & 4 & $5,1 \%$ \\
$20 / 1200$ & 10 & $12,8 \%$ \\
MM & 17 & $21,8 \%$ \\
PL & 12 & $15,4 \%$ \\
NPL & 31 & $39,7 \%$ \\
\hline Total & $\mathbf{7 8}$ & $\mathbf{1 0 0} \%$ \\
\hline
\end{tabular}


Entre las comorbilidades, $25(32,1 \%)$ pacientes presentaron diabetes mellitus y 41 $(52,6 \%)$ hipertensión arterial. Cabe señalar que 16 pacientes presentaron ambas comorbilidades a la vez. La diabetes se presentó en proporciones similares entre mujeres $(29,4 \%)$ y varones $(34,1 \%)$, y entre los distintos grupos etarios. La hipertensión arterial se presentó en proporciones similares entre mujeres $(50 \%)$ y varones $(54,5 \%)$, pero fue significativamente $(p=0,004)$ más frecuente en el grupo etario de 71 a 95 años $(67,6 \%)$, en comparación con los de 51 a 70 años $(57,7 \%)$ y de 18 a 50 años $(21,4 \%)$ (Tabla 3$)$.

Tabla 3. Comorbilidades según edad y sexo de los pacientes con diagnóstico de ceguera irreversible atendidos en el Servicio de Oftalmología General de la Clínica Belén, Coronel Oviedo de 1 de febrero del 2018 al 28 de febrero del 2019

\begin{tabular}{lll}
\hline & \multicolumn{2}{c}{ Comorbilidades } \\
\cline { 2 - 3 } Características generales & $\begin{array}{l}\text { Diabetes mellitus } \\
(\mathbf{n = 2 5 )}\end{array}$ & $\begin{array}{l}\text { Hipertensión arterial } \\
\mathbf{( n = 4 1 )}\end{array}$ \\
\hline $\begin{array}{ll}\mathbf{n}(\mathbf{\%}) \\
\text { Sexo }\end{array}$ & \\
Femenino $(n=34)$ & $10(29,4 \%)$ & $17(50,0 \%)$ \\
Masculino $(n=44)$ & $15(34,1 \%)$ & $24(54,5 \%)$ \\
Grupo etario & & $3(16,7 \%)$ \\
18 a 50 años $(n=18)$ & $3(16,7 \%)$ & $15(57,7 \%)$ \\
51 a 70 años $(n=26)$ & $12(46,2 \%)$ & $23(67,6 \% *)$ \\
71 a 95 años $(n=34)$ & $10(29,4 \%)$ & \\
\hline
\end{tabular}

* Prueba Chi cuadrado: valor $\mathrm{p}=0,004$

La causa más frecuente de ceguera fue glaucoma con 34 pacientes $(43,6 \%)$, la segunda causa fue retinopatía diabética con 19 (24,4\%) pacientes y la tercera causa fue la neuropatía óptica (traumática, compresiva e infecciosa) con $8(10,3 \%)$ pacientes, entre las más frecuentes (Figura 1).

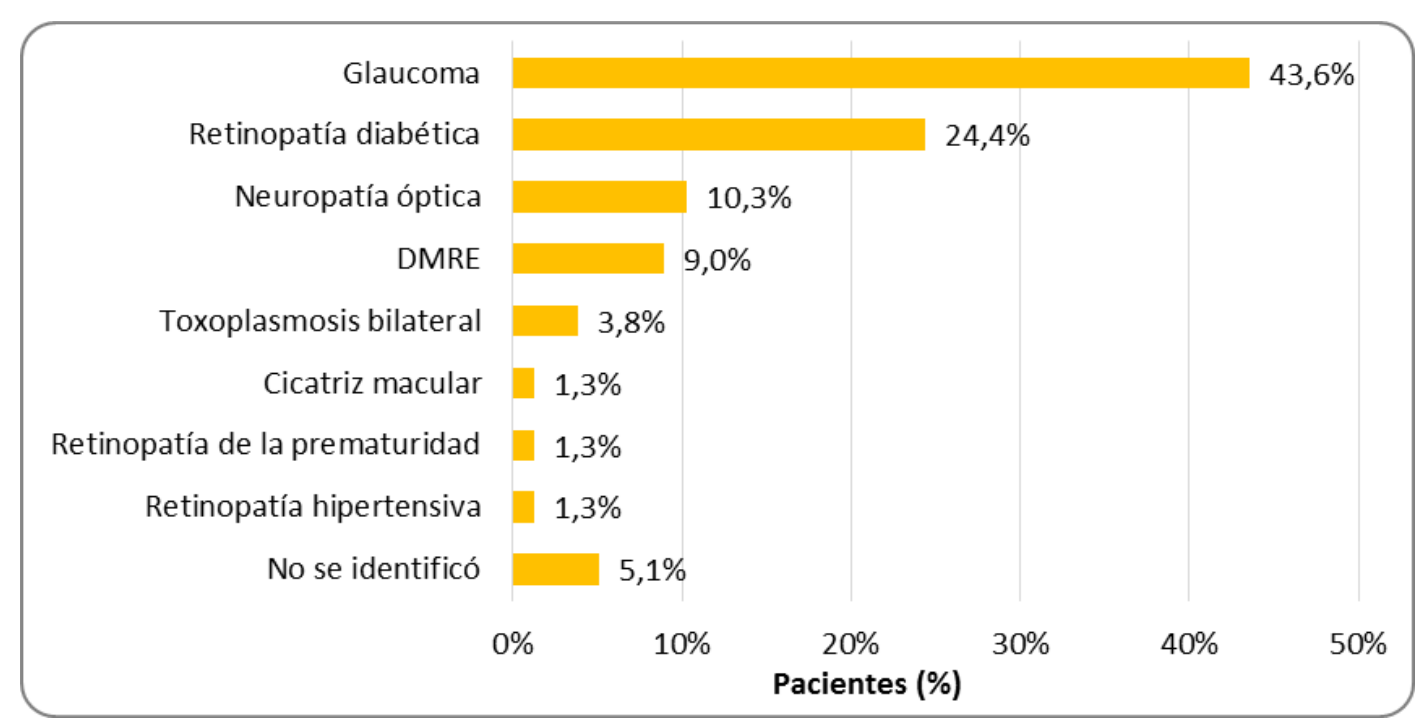

Figura 1. Causas de ceguera en los pacientes con diagnóstico de ceguera irreversible atendidos en el Servicio de Oftalmología General de la Clínica Belén, Coronel Oviedo del 1 de febrero del 2018 al 28 de febrero del 2019

Las causas de ceguera por sexo se presentan en el Figura 2. El 59\% de las mujeres y $32 \%$ de los varones presentaron glaucoma. La retinopatía diabética se presentó levemente mayor en pacientes del sexo masculino (27\%) que en el femenino $(21 \%)$. 
Cabe mencionar que, en un paciente de sexo masculino de 37 años de edad, se encontró cicatriz macular causada por toxoplasmosis en el ojo derecho y ptisis bulbi en el ojo izquierdo; un adolescente de 13 años de edad presentó retinopatía de la prematuridad y una mujer de 79 años de edad presentó hipertensión acelerada maligna, clasificación del grupo 4 de retinopatia hipertensiva maligna.

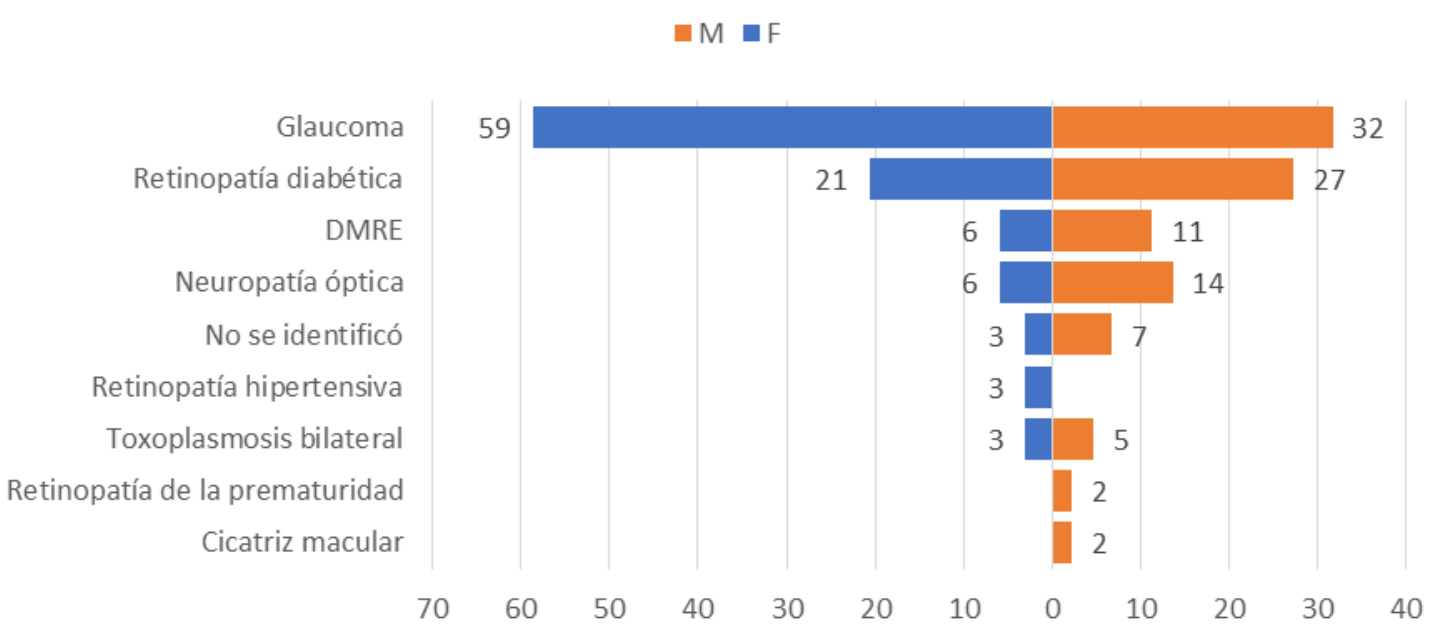

Figura 2. Causas de ceguera según sexo de los pacientes con diagnóstico de ceguera irreversible atendidos en el Servicio de Oftalmología General de la Clínica Belén, Coronel Oviedo del 1 de febrero del 2018 al 28 de febrero del 2019

Tabla 4. Causas de ceguera según grupo etario de los pacientes con diagnóstico de ceguera irreversible atendidos en el Servicio de Oftalmología General de la Clínica Belén, Coronel Oviedo del 1 de febrero del 2018 al 28 de febrero del 2019

\begin{tabular}{|c|c|c|c|c|}
\hline \multirow[t]{2}{*}{ Causas de ceguera legal } & \multicolumn{4}{|c|}{ Grupo etario (años) } \\
\hline & $\begin{array}{c}<18 \\
(n=4) \\
n(\%)\end{array}$ & $\begin{array}{c}18 \text { a } 50 \\
(n=14) \\
n(\%)\end{array}$ & $\begin{array}{c}51 \text { a } 70 \\
(n=26) \\
n(\%)\end{array}$ & $\begin{array}{c}71 \text { a } 95 \\
(n=34) \\
n(\%)\end{array}$ \\
\hline Glaucoma & - & $4(28,6 \%)$ & $13(50,0 \%)$ & $17(50,0 \%)$ \\
\hline Retinopatía diabética & - & $3(21,4 \%)$ & $9(34,6 \%)$ & $7(2,1 \%)$ \\
\hline Neuropatía óptica & $1(25 \%)$ & $4(28,6 \%)$ & $1(3,8 \%)$ & $2(5,9 \%)$ \\
\hline DMRE & - & 0 & $1(3,8 \%)$ & $6(17,6 \%)$ \\
\hline Toxoplasmosis bilateral & $2(50 \%)$ & $1(7,1 \%)$ & - & - \\
\hline Cicatriz macular & - & $1(7,1 \%)$ & - & - \\
\hline $\begin{array}{l}\text { Retinopatía de la } \\
\text { prematuridad }\end{array}$ & $1(25 \%)$ & - & - & - \\
\hline Retinopatía hipertensiva & - & - & - & $1(2,9 \%)$ \\
\hline No se identificó & - & $1(7,1 \%)$ & $2(7,7 \%)$ & $1(2,9 \%)$ \\
\hline
\end{tabular}

Entre los pacientes con Diabetes mellitus, la causa de ceguera más frecuente fue la retinopatía diabética con el $72 \%$ y luego el glaucoma con el $20 \%$. Entre los pacientes que presentaban Hipertensión Arterial, la causa de ceguera más frecuente fue el glaucoma con el $58,5 \%$ Tabla 5 . 
Tabla 5. Causas de ceguera según comorbilidad de los pacientes con diagnóstico de ceguera irreversible atendidos en el Servicio de Oftalmología General de la Clínica Belén, Coronel Oviedo 1 de febrero del 2018 al 28 de febrero del 2019

\begin{tabular}{lllll}
\hline \multirow{2}{*}{ Causas de ceguera legal } & \multicolumn{4}{c}{ Comorbilidad } \\
\cline { 2 - 5 } & \multicolumn{3}{c}{$\begin{array}{c}\text { Diabetes Mellitus } \\
(\mathbf{n = 2 5 )}\end{array}$} & $\begin{array}{c}\text { Hipertensión } \\
\text { Arterial }(\mathbf{n = 4 1})\end{array}$ \\
\cline { 2 - 5 } & $\mathbf{n}$ & $\mathbf{\%}$ & $\mathbf{n}$ & $\mathbf{\%}$ \\
\hline Glaucoma & 5 & $20,0 \%$ & 24 & $58,5 \%$ \\
Retinopatía diabética & 18 & $72,0 \%$ & 10 & $24,4 \%$ \\
Neuropatía óptica & - & - & 0 & $0,0 \%$ \\
DMRE & 2 & $8,0 \%$ & 5 & $12,2 \%$ \\
Toxoplasmosis bilateral & - & - & - & - \\
Cicatriz macular & - & - & - & - \\
Retinopatía de la prematuridad & - & - & - & - \\
Retinopatía hipertensiva & - & - & 1 & $2,4 \%$ \\
No se identificó & - & - & 1 & $2,4 \%$ \\
\hline
\end{tabular}

DMRE: degeneración macular relacionada a la edad

\section{DISCUSIÓN}

De los 78 pacientes con ceguera irreversible incluidos en este estudio, hubo predominio del sexo masculino (56,4\%), entre 51 a 95 años de edad $(76,9 \%)$, y escolaridad primaria incompleta $(41 \%)$. Las características demográficas de los pacientes dependen de la población estudiada (población pediátrica, adulta, o adulta mayor), así en algunos estudios el análisis se realiza en todos los grupos etarios, tal como se hizo en la presente investigación y en dicho análisis se evidencia que la población mayormente afectada por ceguera son los adultos mayores, especialmente los del sexo masculino, en concordancia con otros estudios. Rojas et al. ${ }^{(10)}$ analizaron 1742 registros de paciente atendidos en dos Instituciones Prestadoras de Salud en Medellín y encontraron que los pacientes del sexo masculino eran los que mayormente frecuentaban las consultas oftalmológicas por discapacidad visual $(55,1 \%)$, siendo la edad promedio de los 107 pacientes que analizaron 44,27 años, así mismo, el nivel educativo más frecuente fue el bachillerato completo. Mendoza et al., ${ }^{(14)}$ en el Policlínico "René Vallejo Ortíz" en Cuba estudiaron 47 pacientes ciegos y encontraron que los pacientes del sexo masculino eran los que mayormente tenían ceguera $(55,3 \%)$, así como los adultos mayores ( $\geq 60$ años) $(44,7 \%)$, de manera similar Calero $^{(15)}$ en Nicaragua analizó a pacientes con baja visión en consulta externa de glaucoma y retina, encontró que los pacientes con baja visión tenían 65 años o más $(41,1 \%)$, seguido por aquellos que tenían entre 50 a 64 años $(32,2 \%)$, predominando el sexo masculino $(53,3 \%)$. Gao et al. ${ }^{(13)}$ estudiaron 162 pacientes pediátricos que acudieron a una clínica universitaria de baja visión en China, e identificaron que la mayoría de los pacientes con baja visión eran de sexo masculino, y la edad media de todos ellos fue de 10,73 años.

Dentro de las características clínicas, todos los pacientes incluidos tenían ceguera irreversible, adicionalmente se halló que la agudeza visual tanto del ojo derecho como del ojo izquierdo era la NPL (34,6\% y 39,7\%, respectivamente). En el caso del estudio elaborado por Rojas et al. ${ }^{(10)}$, hallaron que el $17,9 \%$ de los pacientes que analizaron tenían ceguera, puesto que su agudeza visual era NPL, mientras que Mendoza et al. ${ }^{(14)}$, al analizar 47 pacientes ciegos atendidos en el Policlínico "René Vallejo Ortiz", identificaron que la prevalencia de ceguera era de 0,09\%, adicionalmente identificaron que el $33,7 \%$ de los casos eran por causas congénitas.

Calero ${ }^{(15)}$ al analizar la agudeza visual sin corrección de los pacientes encontró que predominaron aquellos con una AV de 20/400 (24,4\%), mientras que con la AV corregida, predominaron los pacientes con 20/80 (30\%). La evaluación visual de los 
pacientes debe ser minuciosa en cada uno de los ojos, considerando las maneras de evaluación actualmente existentes, específicamente dentro de la evaluación de la agudeza visual, por ejemplo para la presente investigación se utilizó en primer lugar la cartilla de Snellen, que es la más utilizada y con dichos resultados se le brindaba al paciente una clasificación según las categorías y subcategorías de la severidad de la deficiencia visual en baja visión y ceguera ${ }^{(10)}$; en quienes no lograban visualizar la presente cartilla, se utilizaban los métodos sin cartilla de optotipos, realizando primero el movimiento de manos, y quienes no percibían dicho movimiento se procedía a la percepción de luz ${ }^{(16)}$.

Sobre las patologías comórbidas, la hipertensión arterial fue la más frecuente, en similares proporciones entre varones y mujeres (54,5\% y $50 \%$, respectivamente) y, mayormente, en aquellos con 71 a 95 años de edad (67,6\%). En el estudio de Rojas et al. ${ }^{(10)}$ predominaron los pacientes con deficiencia visual que presentaban como principal comorbilidad la hipertensión arterial $(31,8 \%)$; así mismo Mendoza et al. ${ }^{(14)}$ identificaron que la patología coexistente más frecuente en los pacientes que analizaron fue la hipertensión arterial $(57,4 \%)$, pero Calero, ${ }^{(15)}$ identificó como principal comorbilidad la diabetes mellitus $(26,7 \%)$.

La presencia de discapacidad visual y/o ceguera, en muchas ocasiones, va a depender de la presencia de comorbilidades que el paciente pueda tener, o tal vez por la clase de actividad diaria que el paciente pueda realizar, y finalmente posiblemente por la falta de evaluaciones clínicas oftalmológicas, sin embargo para ello se requiere que se realicen estudios de tipología prospectiva donde se puedan analizar otras variables que identifiquen otras causas demográficas a las cuales se les pueda atribuir la presencia de estas limitaciones visuales.

Dentro de las causas de la ceguera, se identificó que el $43,6 \%$ de los pacientes incluidos en el estudio tenían como principal etiología el glaucoma, seguido por la retinopatía diabética $(24,4 \%)$. Así mismo, en un estudio nicaragüense, se identificó que la principal causa de la baja visión fue la presencia de glaucoma $(42,2 \%)$, seguido por la retinopatía diabética $(18,9 \%)^{(15)}$; adicionalmente el estudio realizado por Reyes et al. ${ }^{(17)}$, identificó que el glaucoma también fue la principal causa de ceguera $(17,6 \%)$. Ello demuestra que a pesar de que el glaucoma tiene múltiples opciones de tratamiento antes de llegar a la ceguera, este no siempre es aceptado y realizado por la mayoría de los pacientes, conllevando a una pobre adherencia al tratamiento. Esto puede deberse a diversas causas, por lo cual se ve la necesidad de realizar estudios donde se analicen otras variables que puedan influir en este porcentaje de afectados.

Adicionalmente, en el presente estudio se evidenció que la tercera causa de ceguera fue la neuropatía óptica $(10,3 \%)$. En un estudio realizado en Piribebuy, hallaron que la prevalencia de ceguera por la degeneración macular asociada a la edad eran una de las principales causas de baja visión $(11,1 \%)^{(18)}$.

En este estudio, las mujeres padecían mayormente glaucoma $(59 \%)$ y retinopatía diabética $(21 \%)$ y los varones retinopatía diabética $(27 \%)$, DMRE $(11 \%)$ y neuropatía óptica (14\%). Otros estudios hallaron que por lo general son las mujeres quienes presentan alteraciones visuales, deficiencias visuales y/o ceguera ${ }^{(6,17-19)}$.

Se evidenció que el glaucoma era un diagnóstico frecuente en los pacientes mayores de 50 años de edad (50\%), mientras que la retinopatía diabética era una patología frecuente en aquellos pacientes entre 51 a 70 (34,6\%) años de edad, adicionalmente se pudo identificar que el diagnóstico de degeneración macular relacionada a la edad era frecuente en los pacientes entre 71 a 95 años $(17,6 \%)$. Como se evidencia, hay mayor frecuencia de adultos mayores que tienen alguna morbilidad que les genera la ceguera, al respecto Reyes et al.,(16) identificó una mayoría de pacientes mayores de 65 años ciegos.

Por otro lado, se pudo identificar que en aquellos pacientes menores de 18 años las causas de ceguera fueron toxoplasmosis bilateral, neuropatía óptica y retinopatía de la prematuridad. A nivel nacional, Contreras ${ }^{(20)}$ pudo identificar que la principal causa de discapacidad visual en la población pediátrica era la toxoplasmosis bilateral $(28,9 \%)$ seguido por la retinopatía del prematuro (21,5\%), el albinismo (14,9\%) y el nistagmos $(8,3 \%)$, pero dentro de su análisis añadió que la primera causa de baja visión mencionada se presentaba principalmente en pacientes del sexo femenino 
$(51,4 \%)$ y en aquellos que se encontraban en edad escolar $(48,6 \%)$, mientras que la segunda causa mencionada se presentaba mayor en pacientes varones $(53,8 \%)$ y en lactantes (50\%).

Bajo los hallazgos encontrados en esta investigación, se debería concientizar a los profesionales en oftalmología sobre la importancia de realizar evaluaciones clínicas minuciosas en etapas de vida temprana (niños, adolescentes y adultos jóvenes) con la finalidad de disminuir la frecuencia de discapacidad visual moderada, severa y ceguera. Además fomentar la evaluación oftalmológica desde la etapa neonatal, para prevenir los casos de discapacidad visual ocurrida frecuentemente en la etapa adulta mayor, así como realizar campañas de evaluación oftalmológica dirigida a la población en general, para realizar un diagnóstico precoz de las etiologías de la discapacidad visual previniendo de esta manera la ceguera.

Conflicto de intereses: Los autores declaran no tener conflicto de intereses.

Agradecimientos: A la Fundación Visión por permitir la realización de este trabajo de investigación.

Fuente de financiación: No tuvo financiación externa.

Contribución de los autores: Todos los autores han contribuido de igual manera para la investigación y la redacción del presente trabajo.

\section{REFERENCIAS BIBLIOGRÁFICAS}

1. World Health Organization. World report on vision. Suiza: WHO; 2019. URL: https://apps.who.int/iris/bitstream/han dle/10665/328717/9789241516570eng.pdf

2. Ministerio de Salud. Módulo educativo para la promoción de la salud ocular en las instituciones educativas. Documento Técnico. Lima: Dirección General de Intervenciones Estratégicas en Salud Pública; $2017 . \quad$ URL: http://bvs.minsa.gob.pe/local/MINSA/4 198.pdf.

3. Ackland P, Resnikoff S, Bourne R. World blindness and visual impairment: despite many successes, the problem is growing. Community Eye Health. 2017; 30(100): 71-3.

4. Organización Mundial de la Salud. WHO. [Online]; 2018. [Revisado el 24 de Febrero del 2021]. Disponible en: https://www.who.int/es/newsroom/fact-sheets/detail/blindness-andvisual-impairment

5. Arias-Uribe J, Llano-Naranjo $\mathrm{Y}$, Astudillo-Valverde E, Suárez-Escudero J. Caracterización clínica y etiología de baja visión y ceguera en una población adulta con discapacidad visual. Rev Mex Oftalmol. 2018; 92(4): 201-8. DOI: 10.24875/RMO.M18000033.

6. Duerksen $R$, Limburg $H$, Lansingh $V$, Silva J. Review of Blindness and Visual Impairment in Paraguay: Changes Between 1999 and 2011. Ophthalmic Epidemiology. 2013; 20(5): 301-7.

7. Silva J, Mujíca O, Vega E, Barceló A, Lansingh V, Mcleod J, et al. Una evaluación comparativa de la ceguera y la deficiencia visual evitables en siete países latinoamericanos: prevalencia, 8. cobertura y desigualdades. Rev Panam Salud Publica. 2015; 37(1): 21-28.

8. Duerksen R, Limburg $H$, Carron J, Foster A. Cataract blindness in Paraguay - results of a national survey. Ophthalmic Epidemiology. 2003; 10(5): 349-57.

9. National Institutes of Health. NIH. [Online]; 2016. [Revisado el 24 de Febrero del 2021]. Disponible en: https://www.nih.gov/news-

events/news-releases/visual-

impairment-blindness-cases-usexpected-double-2050

10. Rojas S, Ruiz S, Carvajal J, Álvarez M, Duque D, Correa $S$, et al. Caracterización de una población con discapacidad visual (baja visión y ceguera) atendida en dos Instituciones Prestadoras de Salud de Medellín. MEDICINA U.P.B. 2015; 34(1): 30-9.

11. Páez A. Encuesta Nacional de Ceguera y Deficiencia Visual Evitable en Bolivia 2015. RAAB. 2015: 1-21.

12. Fundación Visión. Visión. [Online].; 2021. [Revisado el 24 de Febrero del 2021]. Disponible en: https://www.vision.org.py/

13. Gao G, Manrong Y, Dai J, Feng X, Wang $X$, Zou $L$, et al. Demographic and clinical characteristics of a paediatric low vision population in a low vision clinic in China. Clin Exp Optom. 2016; 99 (3): 274-279. DOI: 10.1111/cxo.12294.

14. Mendoza E, Figueredo L, Figueredo C, Machado A. Prevalencia y algunos aspectos epidemiológicos de la ceguera en el policlínico René Vallejo Ortiz de 
Bayamo. Multimed. 2015; 19(3): 1-9. Disponible en:

http://www.multimedgrm.sld.cu/Docum entos\%20pdf/volumen19-3/13.pdf

15. Calero M. Características clínicasepidemiológicas y factores asociados a la clasificación de grupo funcional de los pacientes con baja visión atendidos en la consulta externa de glaucoma y retina del centro nacional de oftalmología, periodo de Enero a Abril 2015. Tesis. Managua: Universidad Nacional Autónoma de NicaraguaManagua; 2016.

16. Ríos J, Bettin L, Naranjo S, Suárez J, De Vivero C. Pautas para el examen oftalmológico. Enfoque para el estudiante de medicina y el médico general. Universitas Médica. 2017; 58(2): 1-27.

17. Reyes J, Julke R, Matos $Y$, Suárez M, Acosta $M$. Algunas variables epidemiológicas en pacientes débiles visuales y ciegos. Rev Inf Cient. 2015; 90(2): 199-208. Disponible en: https://www.redalyc.org/pdf/5517/551 757251001.pdf

18. Yaacov $F$, Jure $D$, Ocampos J, Samudio M, Furtado J, Carter M, et al. Prevalence and causes of blindness in an urban area of Paraguay. Arq. Bras. Oftalmol. 2012; 75(5): 341-3.

19. Tineo L. Discapacidad visual y ceguera en los pacientes del consultorio de baja visión, Lima 2018. Tesis. Lima: Universidad Nacional Federico Villarreal; 2018.

20. Contreras V. Causas de discapacidad visual en pacientes en edad pediátrica en Asunción, Paraguay. Tesis de segunda especialidad. Asunción: Universidad Católica "Nuestra Señora de la Asunción"; 2011. 